Category: Simulation and Skills competency

\title{
Can online simulation affect medical student's perception of stress?
}

\author{
Scully, A., Nichols, C., Trendall, L., Ong, G.S., Goodson, M. \\ Newcastle University Medicine, Malaysia
}

\section{Background:}

Transition from medical student to junior doctor is challenging and stressful. COVID-19 has restricted student exposure to hospital placements, where students usually begin to develop an approach to coping with future clinical roles. The study aimed to investigate whether online simulation training affected perceptions of stress in medical students in relation to undertaking emergency care when in a simulated on-call setting.

\section{Methods:}

35 randomly selected final year medical students at Newcastle University Medicine Malaysia (NUMed) underwent online surgical simulation training in January 2021 using case scenarios previously piloted in face-to-face teaching. The session simulated a "Bad Day On-Call" for a surgical house officer. Following ethical approval and informed consent, students completed an online Likert scale questionnaire, on their perception of stress before and after simulation training. Questions were related to the on-call experience in general, breaking bad news and clinical decision making. Statistical comparisons of findings between male and female students, before and after training were undertaken using Chi square analysis and Wilcoxon Matched pairs signed ranks test.

\section{Results:}

35 students participated in this online pilot study comprising 21 female and 14 male students. In this study, prior to undertaking simulation training, $61.8 \%$ respondents felt that a surgical on-call was "scary and stressful", $55.9 \%$ students felt that clinical decision making when on-call was stressful, and $52.9 \%$ respondents felt that breaking bad news was stressful. In this study, a significant reduction in perceived stress for on-call in general $(p=0.01)$ and breaking bad news was found $(p=0.04)$. However, there was no significant change for stress surrounding clinical decision making.

\section{Conclusions:}

This study found that online simulation can be used to improve aspects of student's perception of stress in relation to the on-call setting. Online simulation can be a useful tool in supporting students transition to junior doctors in a climate with limited clinical exposure.

Keywords: Stress perception, Online simulation 